\title{
Simulated gravity without true gravity in asymmetric brane-world scenarios
}

\author{
Brandon Carter ${ }^{1}$, Jean-Philippe Uzan ${ }^{2}$, Richard A. Battye ${ }^{3}$ and Andrew Mennim ${ }^{3}$ \\ (1) DARC/LUTH, Observatoire de Paris, 92195 Meudon Cedex, France, \\ (2) Laboratoire de Physique Théorique, UMR 8627 du CNRS, \\ Université de Paris XI, Bâtiment 210, 91405 Orsay Cedex, France, \\ (3) Department of Applied Mathematics and Theoretical Physics, \\ Centre for Mathematical Sciences, University of Cambridge, \\ Wilberforce Road, Cambridge, CB3 0WA, UK
}

11 June 2001

\begin{abstract}
This article investigates asymmetric brane-world scenarios in the limit when the bulk gravity is negligible. We show that, even when true self gravity is negligible, local mass concentrations will be subject to a mutual attraction force which simulates the effect of Newtonian gravity in the non-relativistic limit. Cosmological and also post-Newtonian constraints are examined.
\end{abstract}

\section{Introduction}

Gravity has long been considered to be one of four fundamental forces. Yet it is very different in its nature from the others and, although particle theories have been devised to unify the other three, it has proved surprisingly difficult to unify all four. One possible way to cut this Gordian knot is to consider gravity not as a fundamental force but as a derived one [1]. In this paper we consider one possible way to realise this within the context of higher dimensional theories by explaining gravity as an apparent effect due to the acceleration of a brane-world universe through a five dimensional spacetime.

Following an original suggestion by Randall and Sundrum [2], many authors have considered 5-dimensional scenarios in which effectively 4-dimensional (inverse square law) gravity is induced on a 3-brane hypersurface representing our observed 4-dimensional spacetime due to the effective confinement of the 5-dimensional gravitational field by a strong extrinsic curvature. The kind of cosmological scenario that was originally proposed was characterised by a postulate of $\mathrm{Z}_{2}$ reflection symmetry about the 3-brane hypersurface, in which case the effective 4-dimensional Newton constant $\mathrm{G}_{[4]}$ was found to be proportional to the square of the analogous 5-dimensional gravitational coupling constant $\mathrm{G}_{[5]}$ with a coefficient proportional to the asymptotic limit value $\mathcal{T}_{\infty}$ of the brane tension. A more general relation

$$
\mathrm{G}_{[4]}=\frac{3}{4 \pi \mathcal{T}_{\infty}}\left(\left(\pi^{2} \mathrm{G}_{[5]} \mathcal{T}_{\infty}\right)^{2}-\left(\frac{\bar{f}}{4 \mathcal{T}_{\infty}}\right)^{2}\right),
$$


has been obtained more recently [3] allowing for the possible presence of a force density term $\bar{f}$, such as might arise naturally from a gauge four form coupling 助.

Moreover, for scenarios of this more general reflection symmetry violating kind [5, 6, 7] it has subsequently been shown [9] that it is still possible to recover an effective 4-dimensional Einstein equation for local perturbations on the brane under the same kind of conditions as in the earlier demonstration for the more specialised reflection symmetric case by Shiromizu et al [10]. The applicability of the effective 4-dimensional Einstein equations [9, 10] with the coupling constant given by (1) is restricted to the weak source limit (relevant for the present epoch but not for earlier stages of cosmological evolution during which gravity would have behaved in a "nonconventional" manner [11]) and to the case where the background Weyl tensor contributions, representing the effect of incoming gravitational waves or black holes in the five dimensional "bulk", are sufficiently small.

The purpose of the present work is to consider a very different kind of limit, involving a brane-world scenario in which an inverse square law for gravity is recovered, not by an effective confinement mechanism due to strong extrinsic curvature, but simply by having a sufficiently small five dimensional gravitational coupling constant for genuine gravitation to be entirely negligible. In this weak gravity limit our previous formula (1) will cease to be applicable otherwise an, unacceptable, negative value for $\mathrm{G}_{[4]}$ would be obtained: in the limit considered here, the derivation of (11) for localised perturbations [9] will break down because the Weyl contribution (on whose negligibility its validity depended) will become indeterminate due to $\mathrm{G}_{[5]}$ terms in the denominator. In order to deal with this limit, which is singular from the previous point of view, we need to start again with a fresh approach.

The preceding work 9] shows that the worldsheet geometry, and hence the apparent gravity on the brane, has three origins. There is a first part coming from the geometry of the "bulk" background, a second part arising from the discontinuity of the extrinsic curvature form, $K_{\mu \nu}$, across the worldsheet, and a third part coming from its mean value. In the reflection symmetric case that is most commonly considered, the latter effect is absent (that is, there is a vanishing mean $\left.\left\langle K_{\mu \nu}\right\rangle=0\right)$. The aim of the present article is to investigate the opposite extreme limiting case characterised by the absence of gravitational perturbations and, in particular, of the gravitationally engendered discontinuity of the extrinsic curvature (that is, we will have $\left[K_{\mu \nu}\right]=0$ ) so that it is the mean curvature, identifiable in this case with the value on either side, which provides the dominant contribution.

What will be shown here is that, when five dimensional gravity is absent or negligible, an artificial, and automatically positive, 4-dimensional (inverse square law) gravitation effect will still be present whenever reflection symmetry is broken, with an effective coupling "constant" that will be proportional to the rest frame time component, $K_{00}$ say, of the extrinsic curvature of the averaged background, according to a formula which, instead of (1) will have the form)

$$
\mathrm{G}_{[4]}=\frac{K_{00}^{2}}{4 \pi \mathcal{T}_{\infty}} .
$$

However although it mimics Newtonian gravitation in the non-relativistic limit, the artificial gravitation occurring in the simple type of 5-dimensional 3-brane scenario considered here will produce post-Newtonian effects that will, in general, differ from those of the standard Einstein theory, typically in a parameter range beyond what is observationally admissible.

Although it will be shown that it can be contrived in such a way as to satisfy the cosmological nucleosynthesis constraint and instantaneously mimicking the standard Einstein theory in the 
immediate spacetime neighbourhood of our solar system, the necessary fine tuning would seem to be very artificial. Furthermore, a gravitational "constant" that is proportional to the, possibly variable, extrinsic curvature of the average background can hardly be compatible with the very severe observational limits on deviations from constancy of gravitational coupling.

These considerations lead us to conclude that in its present form the simplest model considered here does not by itself provide a viable alternative theory of gravity. However - quite apart from the possibility that a viable alternative may be provided by some generalisation the simulated worldsheet gravity phenomenon considered here will still be of physical interest and potential practical relevance as a mechanism that can provide small but not necessarily negligible additions to effects of genuinely gravitational origin (in much the same way as, from a laboratory point of view, the genuine gravitational attraction of our Earth is modified by the centrifugal effect of its rotation).

The plan of this article is as follows. The necessary worldsheet perturbation formalism for the general case of a codimension one brane of arbitrary worldsheet dimension $p$ is developed in section 2. The relevant non-gravitating kind of minimally coupled [4] homogeneous isotropic cosmological background to which it will be applied is presented next in section 3, where the first integrated (Friedmann type) form of the dynamical equations is obtained in terms of an adjustable constant of integration (whose interpretation as the energy with respect to the stationary geometry of the external "bulk" is described in the appendix). The heart of this article is in section 4 where it is shown how local perturbations will generically be subject to an attraction of Newtonian gravitational in the non-relativistic limit, and where the more restrictive conditions for agreement with the linearised Einstein equations are derived. The remainder of the discussion is restricted to the cosmologically interesting case of a hypermembrane with worldsheet dimension $p=4$, starting in section 5 where the conditions for compatibility with the standard description of nucleosynthesis are derived. In conclusion, the difficulty of reconciling these global conditions with what is required for matching the parameters governing local gravitational effects is discussed in section 6 .

\section{Lagrangian worldsheet perturbations}

As a technical prerequisite for the perturbation analysis that will be involved, we start by considering the effect of the brane worldsheet perturbation, which we suppose to be generated by an infinitesimal displacement vector field $\xi^{\mu}$ say, on the first and second fundamental forms of the worldsheet, which correspond to tensors denoted by $\bar{\gamma}_{\mu \nu}$ and $K_{\mu \nu}$ using the notation of our previous work [9]. In terms of the background spacetime metric $g_{\mu \nu}$ and the unit normal $\zeta_{\mu}=\nabla_{\mu} \zeta$, where $\zeta$ measures orthogonal distance from the brane (on the side chosen to be considered as positive) the first fundamental tensor is defined simply by

$$
\bar{\gamma}_{\mu \nu} \equiv g_{\mu \nu}-\perp_{\mu \nu}, \quad \perp_{\mu \nu} \equiv \zeta_{\mu} \zeta_{\nu}
$$

In terms of the tangentially projected differentiation operator

$$
\bar{\nabla}_{\mu} \equiv \bar{\gamma}_{\mu}^{\nu} \nabla_{\nu}
$$

the tensor corresponding to the second fundamental form is given円 by

$$
K_{\mu \nu}=-\bar{\nabla}_{\mu} \zeta_{\nu}
$$

\footnotetext{
${ }^{1}$ We are using the MTW sign conventions 12 .
} 
and will automatically have the Weingarten symmetry property $K_{\mu \nu}=K_{\nu \mu}$ as a worldsheet integrability condition. This tensor is also obtainable from the general second fundamental tensor $K_{\mu \nu}{ }^{\rho}$ that was used in an earlier brane perturbation analysis 13 by $K_{\mu \nu}=K_{\mu \nu}{ }^{\rho} \zeta_{\rho}$.

Since our purpose here is to consider the limit in which genuine gravitation is negligible, the geometry will be globally unaffected by the perturbation, so the Eulerian metric perturbation $h_{\mu \nu}=\delta_{\mathrm{E}} g_{\mu \nu}$ that was allowed for in the earlier analysis [13] can simply be set to zero. Thus locally, with respect to coordinates that are comoving with respect to the displacement (so that the brane worldsheet retains the same coordinate locus, $\zeta=0$ ) the only change in the metric tensor will be the purely Lagrangian variation given by the familiar Lie differentiation formula

$$
\delta_{\mathrm{L}} g_{\mu \nu}=2 \nabla_{(\mu} \xi_{\nu)}
$$

using round brackets to indicate index symmetrisation. Preservation of the locus of the worldsheet implies preservation of the direction of the covariant normal $\zeta_{\mu}$, but the change (6) in the metric will entail a corresponding change in amplitude to preserve the unit normalisation property $\zeta_{\mu} \zeta^{\mu}=1$, so it can be seen that the ensuing Lagrangian variation of the unit normal will be given by

$$
\delta_{\mathrm{L}} \zeta_{\mu}=\zeta_{\mu} \zeta_{\nu} \zeta_{\rho} \nabla^{\nu} \xi^{\rho}
$$

This formula can be used to obtain a direct verification of the corresponding Lagrangian variation formula for the first fundamental tensor, namely

$$
\delta_{\mathrm{L}} \bar{\gamma}^{\mu \nu}=-2 \bar{\gamma}_{\sigma}^{(\mu} \bar{\nabla}^{\nu)} \xi^{\sigma}
$$

which is actually valid, not just for the hypermembrane (codimension 1) case under consideration here, but quite generally for a hyperstring (the case with codimension 2 , as in a 6-dimensional world brane scenario [14]) and cases with even higher codimension.

Using the explicit expression

$$
\nabla_{\mu} \zeta^{\nu}=\zeta_{, \mu}^{\nu}+\Gamma_{\mu \rho}^{\nu} \zeta^{\rho}, \quad \Gamma_{\mu \rho}^{\nu}=g^{\nu \sigma}\left(g_{\sigma(\mu, \rho)}-\frac{1}{2} g_{\mu \rho, \sigma}\right),
$$

for the covariant derivative that is involved in (5) and the well known fact that the Lie derivative

$$
\delta_{\mathrm{L}} \Gamma_{\mu \rho}^{\nu}=g^{\nu \sigma}\left(\nabla_{(\rho} \delta_{\mathrm{L}} g_{\mu) \sigma}-\frac{1}{2} \nabla_{\sigma} \delta_{\mathrm{L}} g_{\mu \rho}\right)
$$

of the Christoffel connection can be expressed in terms of the background Riemann tensor $\mathcal{R}_{\mu \nu \rho \sigma}$ as

$$
\delta_{\mathrm{L}} \Gamma_{\mu \rho}^{\nu}=\nabla_{(\mu} \nabla_{\rho)} \xi^{\nu}-\mathcal{R}^{\nu}{ }_{(\mu \rho) \sigma} \xi^{\sigma} .
$$

Together with (7) and (8), this enables us to calculate the corresponding variation of the extrinsic curvature as

$$
\delta_{\mathrm{L}} K^{\mu \nu}=\zeta_{\rho} \bar{\gamma}_{\kappa}^{(\mu} \bar{\gamma}_{\lambda}^{\nu)}\left(\bar{\nabla}^{(\kappa} \bar{\nabla}^{\lambda)} \xi^{\rho}+\mathcal{R}_{\sigma \rho \lambda}^{\kappa \rho} \xi^{\sigma}\right)+4 K_{\sigma}^{(\mu} \bar{\nabla}^{\nu)} \xi^{\sigma} .
$$

In the language of [13], this can be obtained from the formula $K_{\mu \nu}=K_{\mu \nu}{ }^{\rho} \zeta_{\rho}$ using (7) and the general expression for $\delta_{\mathrm{L}} K_{\mu \nu}{ }^{\rho}$. If one makes the convenient choice of an orthogonal gauge, setting

$$
\xi^{\mu}=\xi \zeta^{\mu},
$$


it can be seen that (12) will be expressible directly in terms of the scalar displacement amplitude $\xi$ as

$$
\delta_{\mathrm{L}} K^{\mu \nu}=\bar{\gamma}_{\kappa}^{(\mu}{ }_{\kappa} \bar{\gamma}_{\lambda}^{\nu}\left(\bar{\nabla}^{(\kappa} \bar{\nabla}^{\lambda)} \xi+\mathcal{R}_{\sigma \rho}^{\kappa \rho \lambda} \perp_{\rho}^{\sigma} \xi\right)+3 K^{\sigma \mu} K_{\sigma}^{\nu} \xi .
$$

In what follows we shall be particularly interested in the trace $K=K_{\nu}^{\nu}$, for which the corresponding variation can be seen to be expressible in terms of the background Ricci tensor, $\mathcal{R}_{\mu \nu}=\mathcal{R}_{\sigma \mu}{ }^{\sigma}{ }_{\nu}$, as

$$
\delta_{\mathrm{L}} K=\bar{\nabla}^{\mu} \bar{\nabla}_{\mu} \xi+\left(K^{\mu \nu} K_{\mu \nu}+\perp^{\mu \nu} \mathcal{R}_{\mu \nu}\right) \xi .
$$

This formula leads to a noteworthy cancellation when used in conjunction with the contraction of the well known Gauss identity (see, for example, the preceding analysis 15]) according to which the Ricci scalar $\bar{R}$ of the induced metric on the hypersurface will be given by

$$
\bar{R}=K^{2}-K^{\mu \nu} K_{\mu \nu}+2 \bar{\gamma}^{\mu \nu} \mathcal{R}_{\mu \nu}-\mathcal{R} .
$$

The preceding formulae are valid regardless of the dimension, $p$ say, of the worldsheet under consideration, the only restriction being that it should have codimension one. However we must take account of this dimension in the next step, which depends on separating the trace-free part of the extrinsic curvature tensor,

$$
C_{\mu \nu}=K_{\mu \nu}-\frac{1}{p} K \bar{\gamma}_{\mu \nu}, \quad C_{\nu}^{\nu}=0 .
$$

and also the trace-free part of the background Ricci tensor,

$$
\mathcal{S}_{\mu \nu}=\mathcal{R}_{\mu \nu}-\frac{1}{p+1} \mathcal{R} g_{\mu \nu}, \quad \mathcal{S}_{\nu}^{\nu}=0
$$

which vanishes whenever the background satisfies Einstein type vacuum equations, with or without a cosmological constant. What is remarkable is that the form of (15) is such that the identity (16) can be used to eliminate not just the extrinsic curvature trace $K$, but also the background Ricci trace scalar, $\mathcal{R}$, in favour just of the intrinsic Ricci trace scalar $\bar{R}$, and hence

$$
\delta_{\mathrm{L}} K=\bar{\nabla}^{\mu} \bar{\nabla}_{\mu} \xi+\frac{\xi}{p-1}\left(\bar{R}+p C^{\mu \nu} C_{\mu \nu}-(p+1) \bar{\gamma}^{\mu \nu} \mathcal{S}_{\mu \nu}\right)
$$

This generalises the results by Garriga and Vilenkin [16]. The particular case $p=2$ of this identity applies to any ordinary 2-surface in 3-dimensional space, while the case $p=3$ applies to the worldsheet of an ordinary membrane, including the case of a cosmological domain boundary, in 4-dimensional spacetime. What we shall be concerned with here is the case $p=4$ of a 3 -brane in a 5-dimensional spacetime background. In this latter context, the significant feature of (19) is that in typical brane-world scenarios the quantities in the bracket on the right are all zero or tend to zero at late cosmological times, unlike the cancelled traces $K$ and $\mathcal{R}$, whose amplitudes remain very large.

\section{Homogeneous cosmological reference configuration}

The previous analysis will allow us to study the Newtonian limit of the theory. First, we shall study homogeneous and isotropic reference configuration which will also be of interest for the 
cosmological solutions. Our purpose now is thus to consider the case of a hypermembrane governed by an equation of motion taking the standard form

$$
\overline{\mathcal{T}}^{\mu \nu} K_{\mu \nu}=\bar{f},
$$

that applies 15] in the limit when self gravitation is negligible so that the background geometry can be considered to be smooth. In this dynamical equation, $\bar{f}$ is the orthogonal component of the external force density, and $\overline{\mathcal{T}}^{\mu \nu}$ is the surface stress energy density tensor, which will satisfy an intrinsic pseudo-conservation law of the form

$$
\bar{\gamma}_{\nu \rho} \bar{\nabla}_{\mu} \overline{\mathcal{T}}^{\mu \nu}=0
$$

provided the external force density has no tangential component. We shall restrict our attention

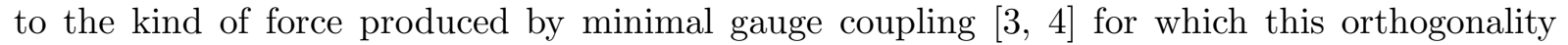
condition will automatically be satisfied, and for which the force density $\bar{f}$ will simply be a constant.

We wish to study perturbations of a homogeneous isotropic reference configuration in a background satisfying Einstein type vacuum equations of the standard form

$$
\mathcal{S}_{\mu \nu}=0
$$

As discussed in the preceding work [3], application of the relevant generalisation [8] of the well known 4-dimensional Birkhoff theorem [12] allows us to conclude that the background metric will have the static form

$$
\mathrm{d} s^{2}=r^{2} \mathrm{~d} \ell^{2}+\frac{\mathrm{d} r^{2}}{\mathcal{V}}-\mathcal{V} \mathrm{d} t^{2}
$$

where $\mathrm{d} \ell^{2}$ is the positive-definite space metric of a $(p-1)$-dimensional sphere, plane, or antisphere with constant curvature, $k$ say, respectively positive, zero, or negative, and $\mathcal{V}$ is a function only of $r$. The location of the brane worldsheet will be given by an expression of the form $r=a$ where $a$ is a function only of $t$. There will be a equivalent brane based formulation

$$
\mathrm{d} s^{2}=r^{2} \mathrm{~d} \ell^{2}+\mathrm{d} \zeta^{2}-\nu^{2} \mathrm{~d} \tau^{2}
$$

in which the worldsheet locus is given simply by $\zeta=0$, and in which $\tau$ represents proper time on this locus, so that $\nu=1$ there. Using a dot to denote partial differentiation with respect to $\tau$, and a dash for partial differentiation with respect to $\zeta$, the rate of change of the scale factor $a$ will satisfy the conditions

$$
\dot{a}^{2}=r^{\prime 2}-\mathcal{V} \quad \text { and } \quad \dot{a}^{2}=\dot{r}^{2} / \nu^{2} .
$$

In such a system, the first fundamental form (3) specifying the induced metric on the worldsheet, is

$$
\mathrm{d} \bar{s}^{2}=\bar{\gamma}_{\mu \nu} \mathrm{d} x^{\mu} \mathrm{d} x^{\nu}=a^{2} \mathrm{~d} \ell^{2}-\mathrm{d} \tau^{2},
$$

and the corresponding expression for second fundamental form (5) is

$$
K_{\mu \nu} \mathrm{d} x^{\mu} \mathrm{d} x^{\nu}=-a r^{\prime} \mathrm{d} \ell^{2}+\nu^{\prime} \mathrm{d} \tau^{2},
$$

which can also be expressed as

$$
K_{\mu \nu}=-\frac{r^{\prime}}{a} \bar{\gamma}_{\mu \nu}+\left(\nu^{\prime}-\frac{r^{\prime}}{a}\right) \bar{u}_{\mu} \bar{u}_{\nu} .
$$


in terms of the preferred timelike unit vector $\bar{u}_{\nu} \equiv \nabla_{\nu} \tau$ that specifies the cosmological reference frame. In terms of this unit vector, the stress-energy momentum tensor will have the isotropic perfect fluid form

$$
\overline{\mathcal{T}}^{\mu \nu}=-\mathcal{T} \bar{\gamma}^{\mu \nu}+(\mathcal{U}-\mathcal{T}) \bar{u}^{\mu} \bar{u}^{\nu},
$$

where $\mathcal{U}$ is the total rest frame energy density and $\mathcal{T}$ is the total brane tension. It can be deduced from (27) that the dynamical equation (20) takes the form

$$
\mathcal{U} \nu^{\prime}+(p-1) \mathcal{T} \frac{r^{\prime}}{a}=\bar{f}
$$

from which we deduce that the extrinsic curvature scalar is given by

$$
K=-\frac{\bar{f}}{\mathcal{U}}-(p-1)\left(1-\frac{\mathcal{T}}{\mathcal{U}}\right) \frac{r^{\prime}}{a},
$$

while the trace-free part will have the form

$$
C_{\mu \nu}=C\left(\frac{1}{p} \bar{\gamma}_{\mu \nu}+\bar{u}_{\mu} \bar{u}_{\nu}\right),
$$

so that the scalar in (19) is explicitly given by

$$
C^{2}=\frac{p}{p-1} C^{\mu \nu} C_{\mu \nu}=\left(\nu^{\prime}-\frac{r^{\prime}}{a}\right)^{2} .
$$

Due to homogeneity, the non-trivial content of the pseudo-conservation law (21) reduces to the simple energy equation

$$
\dot{\mathcal{U}}+(p-1)(\mathcal{U}-\mathcal{T}) \frac{\dot{a}}{a}=0 .
$$

Using (34) and the relation $\dot{r}^{\prime}=\nu^{\prime} \dot{a}$ obtained from (25), the dynamical equation (30) can be rewritten as

$$
\frac{\mathrm{d}}{\mathrm{d} \tau}\left(\mathcal{U} r^{\prime} a^{p-1}\right)=\frac{\bar{f}}{p} \frac{\mathrm{d}}{\mathrm{d} \tau}\left(a^{p}\right)
$$

Since, as remarked above, the minimal coupling postulate automatically ensures [3, 迆 that $\bar{f}$ is constant, this equation will be immediately integrable to give

$$
r^{\prime}=\frac{a}{\mathcal{U}}\left(\frac{\bar{f}}{p}+\frac{E_{\mathrm{B}}}{a^{p}}\right),
$$

where $E_{\mathrm{B}}$ is a constant of integration, which can be interpreted as being proportional to the global energy of the system. Since the concept of energy normally involves reference to some stationary background reference system so as to be well defined, it can not be used in the usual global cosmological context of an expanding universe model. It will, however, be shown in the appendix how a bulk background which is static — due to the generalised Birkhoff theorem [8] - will allow us to define this energy in an unambiguous manner for minimally coupled scenarios of the kind considered here. Using (30) again, we obtain the corresponding formula

$$
\nu^{\prime}=\frac{\bar{f}}{\mathcal{U}}-(p-1) \frac{\mathcal{T}}{\mathcal{U}^{2}}\left(\frac{\bar{f}}{p}+\frac{E_{\mathrm{B}}}{a^{p}}\right),
$$


which completes the evaluation of the coefficients in the expression (27) for the second fundamental form. Therefore, the extrinsic curvature scalar is given by

$$
K=-\frac{\bar{f}}{p \mathcal{U}}\left(1+(p-1) \frac{\mathcal{T}}{\mathcal{U}}\right)-\frac{p-1}{\mathcal{U}}\left(1-\frac{\mathcal{T}}{\mathcal{U}}\right) \frac{E_{\mathrm{B}}}{a^{p}},
$$

and the corresponding expression for the scalar coefficient $C$ in (32) and (33) is

$$
C=\frac{(p-1) \bar{f}}{p \mathcal{U}}\left(1-\frac{\mathcal{T}}{\mathcal{U}}\right)-\frac{1}{\mathcal{U}}\left(1+(p-1) \frac{\mathcal{T}}{\mathcal{U}}\right) \frac{E_{\mathrm{B}}}{a^{p}} .
$$

As in the preceding work [3, 4] on the self gravitating case, we adopt the usual supposition that the hypersurface stress energy tensor $\overline{\mathcal{T}}^{\mu \nu}$ in (20) has the form

$$
\overline{\mathcal{T}}^{\mu \nu}=\mathcal{T}_{\infty} \bar{\gamma}^{\mu \nu}+\bar{\tau}^{\mu \nu}
$$

in which $\bar{\tau}^{\mu \nu}$ is the part attributable to matter fields on the brane and $\mathcal{T}_{\infty}$ is a constant interpretable as the "bare" brane tension meaning the limit to which the tension and energy density tend in the infinitely distended limit. In the homogeneous case characterised by (29) the material contribution will be given in terms of average cosmological density and pressure functions

$$
\bar{\rho}=\mathcal{U}-\mathcal{T}_{\infty}, \quad \bar{P}=\mathcal{T}_{\infty}-\mathcal{T},
$$

by

$$
\bar{\tau}^{\mu \nu}=\bar{P} \bar{\gamma}^{\mu \nu}+(\bar{\rho}+\bar{P}) \bar{u}^{\mu} \bar{u}^{\nu} .
$$

In the large expansion limit $a \rightarrow \infty$ that is relevant at late cosmological times the material contributions will tend to zero, $\bar{\rho} \rightarrow 0$ and $\bar{P} \rightarrow 0$, so that $\mathcal{U} \rightarrow \mathcal{T}_{\infty}$ and $\mathcal{T} \rightarrow \mathcal{T}_{\infty}$, which implies that we shall have

$$
K \rightarrow K_{\infty} \equiv-\frac{\bar{f}}{\mathcal{T}_{\infty}}, \quad C \rightarrow 0,
$$

which will be discussed in more details in the following sections.

\section{Perturbed Configuration}

We now turn our attention to an "actual" configuration in which the brane worldsheet deviates from that of the homogeneous reference configuration described above by a small displacement with amplitude $\xi$ of the kind described in section 2. Using a tilde to distinguish perturbed quantities from their analogues in the reference configuration, we take the first and second fundamental tensors of the perturbed worldsheet to be given, using the notation of (11) and (12), by

$$
\widetilde{\bar{\gamma}}_{\mu \nu}=\bar{\gamma}_{\mu \nu}+\delta_{\mathrm{L}} \bar{\gamma}_{\mu \nu}
$$

and

$$
\widetilde{K}_{\mu \nu}=K_{\mu \nu}+\delta_{\mathrm{L}} K_{\mu \nu} .
$$

The corresponding perturbed version of the equation of motion (20) will have the form

$$
\widetilde{\overline{\mathcal{T}}}^{\mu \nu} \widetilde{K}_{\mu \nu}=\bar{f}
$$


with a perturbed surface energy-momentum tensor of the form

$$
\widetilde{\overline{\mathcal{T}}}^{\mu \nu}=-\mathcal{T}_{\infty} \widetilde{\bar{\gamma}}^{\mu \nu}+\widetilde{\bar{\tau}}^{\mu \nu}
$$

in which the constants $\mathcal{T}_{\infty}$ and $\bar{f}$ are just the same as in the reference configuration.

By subtracting (46) from its unperturbed analogue (20) we are left with a dynamical source equation for $\xi$ having the form

$$
\delta_{\mathrm{L}} K=K_{\mu \nu}\left(\widetilde{\epsilon}^{\mu \nu}-\epsilon^{\mu \nu}\right)+\epsilon^{\mu \nu} \delta_{\mathrm{L}} K_{\mu \nu},
$$

with $\delta_{\mathrm{L}} K$ as given by (19) and where we have introduced the recalibrated dimensionless material stress-energy contribution

$$
\epsilon^{\mu \nu} \equiv \frac{1}{\mathcal{T}_{\infty}} \bar{\tau}^{\mu \nu}
$$

When $\epsilon_{\mu \nu}=0$ and $\widetilde{\epsilon}_{\mu \nu}=0$, equation (48) can be interpreted, using (19), as the equation of evolution for a scalar field $\xi$ non-minimally coupled to the Ricci scalar, $\bar{R}$, and with an effective mass given (of the kind discussed by Garriga and Vilenkin [16]) by

$$
m_{\xi}^{2}=-\frac{1}{p-1}\left(p C^{\mu \nu} C_{\mu \nu}-(p+1) \bar{\gamma}^{\mu \nu} \mathcal{S}_{\mu \nu}\right) .
$$

\subsection{Simulation of Newtonian gravity}

Let us now consider the field in the neighbourhood of a concentrated non-relativistic source, that is one whose energy-momentum contribution is given in terms of a preferred timelike unit reference vector $\bar{u}^{\mu}$ by the approximate formula

$$
\widetilde{\bar{\tau}}^{\mu \nu}-\bar{\tau}^{\mu \nu} \simeq \delta \rho \bar{u}^{\mu} \bar{u}^{\nu}
$$

We consider a configuration that deviates from an almost uniform and low curvature reference configuration by the presence of a matter distribution within a region which is small compared to the reference curvature scale, so that we can work at first order both in $\epsilon_{\mu \nu}$ and in the displacement $\xi$. Hence, the second term of the right hand side of (48) is of order $\mathcal{O}(\epsilon \xi)$ and will thus be negligible compared with the first term. On short enough lengthscales, we can keep only the gradient terms of highest order, so that we have that $\delta_{\mathrm{L}} K \simeq \bar{\square} \xi$ and the preceding source equation for $\xi$ will reduce to the simple form

$$
\bar{\square} \xi \simeq \frac{K_{00}}{\mathcal{T}_{\infty}} \delta \rho
$$

using the notation

$$
\bar{\square} \equiv \bar{\nabla}^{\mu} \bar{\nabla}_{\mu}, \quad K_{00}=K_{\mu \nu} \bar{u}^{\mu} \bar{u}^{\nu} .
$$

This approximation is the same as the one used when one neglects the cosmological fluids with respect to the mass of the Sun on length scales of order of the Solar system.

The operator $\bar{\square}$ is interpretable as the wave operator of the local spacetime geometry on the brane, and $K_{00}$ is the time component of the extrinsic curvature with respect to the preferred rest frame, which is interpretable simply as the component of its acceleration orthogonal to the worldsheet. In terms of $p$-dimensional worldsheet coordinates $\sigma^{i}$, with respect to which the 
induced metric has components $\gamma_{i j}$ and determinant $\|\gamma\|$, (53) can be replaced by the more explicit expressions

$$
\bar{\square} \xi=\|\gamma\|^{-1 / 2}\left(\|\gamma\|^{1 / 2} \gamma^{i j} \xi_{, i}\right)_{, j}, \quad K_{00}=K_{i j} \bar{u}^{i} \bar{u}^{j} .
$$

Using (5), (8) and (13), the resulting variation of the first fundamental tensor $\bar{\gamma}_{\mu \nu}$ can be seen to be

$$
\delta_{\mathrm{L}} \bar{\gamma}_{\mu \nu}=-2 K_{\mu \nu} \xi
$$

Since, on the local scale under consideration, the unperturbed intrinsic metric can be taken to be of flat Minkowski form, $\gamma_{i j}=\eta_{i j}$ say, the corresponding perturbed form of the induced metric will be expressible in the form

$$
\widetilde{\gamma}_{i j}=\eta_{i j}+h_{i j}
$$

in which, by (55), the induced metric perturbation will be given by

$$
h_{i j}=-2 K_{i j} \xi
$$

For a spherical or hyperspherical static source distribution with integrated mass $\widetilde{M}$, the equation (52) for $\xi$ has the well known solution

$$
\xi=\left(\frac{-K_{00}}{(p-3) \Omega^{[p-2]} \mathcal{T}_{\infty}}\right) \frac{\widetilde{M}}{\bar{r}^{p-3}},
$$

for $p \geq 4$, while for the case of a circularly symmetric source distribution on a membrane in an ordinary 4-dimensional spacetime background the solution is

$$
\xi=\frac{K_{00}}{2 \pi \mathcal{T}_{\infty}} \widetilde{M} \ln \bar{r}
$$

where, in both cases, $\bar{r}$ is the radial distance from the centre.

The rest frame component

$$
h_{00}=h_{i j} u^{i} u^{j}
$$

of this perturbation is given in terms of the corresponding rest frame component $K_{00}$ of the second fundamental form by the dimensionally generalised Newtonian limit formula

$$
h_{00}=2 \mathrm{G}_{[p]} \frac{\widetilde{M}}{\bar{r}^{p-3}},
$$

if one identifies the unrationalised $p$-dimensional gravitational constant as

$$
\mathrm{G}_{[p]}=\frac{K_{00}^{2}}{(p-3) \Omega^{[p-2]} \mathcal{T}_{\infty}} \quad(p>3) .
$$

We now concentrate on the constraints that have to be taken into account in the case $p=4$ which is of greatest interest to us but we will also we then discuss some applications of the $p=3$ case. 


\subsection{Observational constraints when $p=4$}

It seems from (60) and (62) that this mechanism can provide a plausible theory for Newtonian gravitation but there are some important additional constraints that have to be taken into account. In this section we shall start by considering the constraint arising from the requirement that as well as reproducing Newtonian effects, the theory should also have the correct relativistic behaviour in the weak-field limit, meaning that it should for instance agree with solar system measurements of gravity.

With respect to an unperturbed metric of the standard Minkowski form, $\eta_{00}=-1, \eta_{0 a}=0$, $\eta_{a b}=\delta_{a b}$ (using Latin space indices, $a=1,2,3$ ) a perturbation $h_{i j}$ of the form introduced in (56) will be expressible in terms of the relevant parameters of standard post-Newtonian parameterisation [17] by the formulae

$$
\begin{aligned}
& h_{00} \simeq 2 U-2 \beta U^{2}, \\
& h_{0 a} \simeq-\frac{1}{2}\left(\alpha_{1}-2 \alpha_{2}\right) w_{a} U, \\
& h_{a b} \simeq 2 \gamma U \delta_{a b}
\end{aligned}
$$

so that the trace $h=h_{i}^{i}$ will be given by the formula

$$
h=2(3 \gamma-1) U+2 \beta U^{2},
$$

in which the quantities $w_{a}$ are the velocity components of the PPN coordinate system relative to the mean rest-frame of the universe (which is usually interpreted to be that of the cosmic microwave background) and $U$ is the Newtonian potential, whose asymptotic form at large distances from the source will of course be given by $U \sim \mathrm{G}_{[4]} \tilde{M} / \bar{r}$. The standard post-Newtonian parameters $\gamma, \beta, \alpha_{1}$ and $\alpha_{2}$ are fixed physical constants.

Any gravitational theory should predict values for these constants and confront the results with what is observed. In particular, Einstein's general relativity gives that $\gamma=1, \beta=1$ and that all other post-Newtonian parameters must be zero. Less restrictively, Jordan - Brans Dicke theory involves a free parameter $\omega$ in terms of which $\gamma=(\omega+1) /(\omega+2)$, while giving $\beta=1$ and zero values for the other post-Newtonian parameters 18 . The freedom to adjust the parameter $\omega$ can be used [19] to match the the Jordan-Brans-Dicke theory to a generic configuration of the form (57). However this freedom ceases to exist when measurements of gravitational light deflection, and the Shapiro time delay effect, are taken into account. These effects provide an observational bound on the PPN parameter $\gamma$ whose current value is given by

$$
|\gamma-1|<3 \times 10^{-4}
$$

This quantity measures how much space-curvature is produced by a unit rest mass, whereas the quantity $\beta$ measures the degree of "non-linearity" in the superposition law of gravity, an effect whose estimation is beyond the scope of the purely linear analysis provided here. By substituting (57) in (63) and (64) it can be seen that what our linear analysis does provide is a limit

$$
\left|\frac{K}{K_{00}}-2\right|<9 \times 10^{-4}
$$

that must somewhow be satisfied by the extrinsic curvature as a condition for compatibility with the observational limit (65) on the assumption that the unperturbed configuration is spatially isotropic. 
Apparent deviations from isotropy might arise from the effects of the parameters $\alpha_{1}$ and $\alpha_{2}$, which (due mainly to Lunar ranging experiments) are subject to constraints [17] given by

$$
\alpha_{1}<2 \times 10^{-4}, \quad \alpha_{2}<4 \times 10^{-7} .
$$

In order to be compatible with these limits also, in addition to satisfying the limit (66), the unperturbed extrinsic curvature must satisfy the restriction

$$
\frac{K_{0} a}{K_{00}} \approx-\frac{1}{4} \alpha_{1} w_{a}
$$

with $\alpha_{1}$ subject to (67) where the velocity vector $w_{a}$ has magnitude of the order of $200 \mathrm{~km} \mathrm{~s}^{-1}$ while its direction is opposite to that of the cosmic microwave background relative to the Earth.

Before considering the further restrictions imposed by global cosmological considerations on this model for effective gravity in a brane-world scenario, we shall briefly digress to discuss analogous local effects in 2-branes.

\subsection{Application to the case $p=3$}

As a second application, consider the well known "museum" experiment used to illustrate how gravity is a consequence of spacetime geometry and in which a heavy bead of mass $M$ is placed on a piece of fabric of tension, $\mathcal{T}_{\infty}$ say, in the local terrestrial gravitational acceleration field with magnitude $g$. The weight of the heavy bead produces a dip in the level of the surrounding fabric, and thus creates an effective potential well in which another lighter bead may be observed to orbit the central point of attraction in much the same way as a planet orbits the Sun, but with an acceleration that varies inversely as the distance, not inversely as the square of the distance as in the Keplerian planetary case. In this example, at lowest order the component of the force density orthogonal to the worldsheet (vertical in this case) is

$$
\bar{f}=\mathcal{T}_{\infty} g
$$

and the corresponding displacement will be given by

$$
\xi=\frac{g}{2 \pi \mathcal{T}_{\infty}} M \ln r
$$

so that the effective gravitational potential is

$$
V_{\text {eff }}=\frac{g^{2}}{2 \pi \mathcal{T}_{\infty}} M \ln r
$$

as long as we are sufficiently far from the centre for the weak-field regime description to be applicable. On approaching the centre, on enters a strong-field regime.

A less trivial but much more speculative application is implicitly suggested by the recent proposal [20] that the rotation curve of spiral galaxies may be explained by supposing that these galaxies are trapped on lightweight domain wall, of which the thickness is comparable with the thickness of the luminous part of the galaxy. The idea of Dvali et al is that the domain wall supports a massless scalar particle zero mode confined to $(2+1)$-dimensions with a coupling to 
the galactic matter so that at distances larger than the typical thickness of the wall the total potential for the net force that acts will have an extra logarithmic contribution. The formula

$$
v^{2}=r \frac{\mathrm{d} V}{\mathrm{~d} r} .
$$

can be used to obtain an observational estimate of the radial dependence of the effective gravitational potential $V$ from measurements of the galactic rotation velocity $v$. In typical cases the result can be described in terms of a plateau at distances larger than the radius of the galaxy disk where $v$ tends towards a constant limit $v_{\infty}$ so that one obtains an empirical formula of the form

$$
V=-\frac{\mathrm{G} M_{\mathrm{Gal}}}{r}+v_{\infty}^{2} \ln r,
$$

with $v$ ranging from $10^{-4}$ to $10^{-3}$ in the relativistic units we are using, that is $v_{\infty}$ ranges from $60 \mathrm{~km} \mathrm{~s}^{-1}$ to $300 \mathrm{~km} \mathrm{~s}^{-1}$. It was suggested in [20] that these observations be used to tune the parameters, such as the energy scale, of their light scalar field, which does not imply any cosmological catastrophe.

What we wish to point out here is that this scalar field mechanism is very similar in its effect to the simulated gravity mechanism we have been describing here. According to our preceding analysis, if the galaxies were constrained to move on a 2-brane subject to an acceleration corresponding to a local extrinsic curvature component $K_{00}$ then, in addition to the genuinely gravitational inverse square law contribution, there would be an extra contribution to the effective gravitational field so that the effective gravitational potential would be given by

$$
V_{\mathrm{eff}}=-\frac{\mathrm{G} M_{\mathrm{Gal}}}{r}+\frac{K_{00}^{2}}{\pi \mathcal{T}_{\infty}} M_{\mathrm{Gal}} \ln r
$$

This would evidently have the same effect as the potential (73) if the acceleration and tension of the membrane satisfy

$$
\frac{K_{00}^{2}}{\mathcal{T}_{\infty}}=\frac{\pi v_{\infty}^{2}}{M_{\text {Gal }}}
$$

Of course, in order for this variant of the original mechanism to be made astrophysically plausible, it would still be necessary to find some natural mechanism for actually trapping the galaxies on the 2-brane.

\section{Cosmological matching conditions.}

\subsection{Effective Friedmann equation}

It should be remarked that none of the preceding results depend on the specific functional form

of the background metric coefficient $\mathcal{V}$ in (23). The most general solution, as derived in [\&] and discussed in the preceding work [3, 9] is

$$
\mathcal{V}=k-\frac{2 \Lambda}{p(p-1)} r^{2}-\frac{2 \mathrm{G}_{[p+1]} \mathcal{M}}{r^{p-2}}
$$

The first term is the curvature, $k$, of the homogeneous isotropic space metric $\mathrm{d} \ell^{2}$, the second term is the effect of the cosmological constant, $\Lambda$, of the bulk and the third term is the contribution 
of the total asymptotic mass, $\mathcal{M}$. Substituting this in (25), and using the solution (36) of the worldsheet evolution equation (20), we immediately obtain the relevant Friedmann type equation for the comoving lengthscale $r=a$ of the brane in the form

$$
\left(\frac{\dot{a}}{a}\right)^{2}=\frac{1}{\mathcal{U}^{2}}\left(\frac{\bar{f}}{p}+\frac{E_{\mathrm{B}}}{a^{p}}\right)^{2}+\frac{2 \Lambda}{p(p-1)}+\frac{2 \mathrm{G}_{[p+1]} \mathcal{M}}{a^{p}}-\frac{k}{a^{2}} .
$$

As observational evidence suggests that the spatial curvature of the universe does not play a significant role, we shall assume that $k=0$ from now on. When the gravitational coupling is very small, the asymptotic mass would have to be very large to have a significant effect so we shall also set $\mathcal{M}=0$. However, in the case where there is no gravity, this term could be reinstated with a different physical interpretation.

In order to match our model to what is observationally known about the large-scale structures of our actual universe, an obvious first step is to set the brane worldsheet dimension to $p=4$. As in the preceding discussion [3] of the gravitationally coupled case, the low density epoch in which we exist can be dealt with by an expansion in powers of the dimensionless density ratio

$$
\varepsilon=\frac{\bar{\rho}}{\mathcal{T}_{\infty}}
$$

Equation (77) can be rewritten as

$$
\left(\frac{\dot{a}}{a}\right)^{2}=\frac{\bar{\Lambda}_{4}}{3}+\frac{1}{\mathcal{T}_{\infty}^{2}}\left[\frac{1}{(1+\varepsilon)^{2}}\left(\frac{\bar{f}}{4}+\frac{E_{\mathrm{B}}}{a^{4}}\right)^{2}-\left(\bar{f} \frac{\bar{f}}{4}\right)^{2}\right]
$$

where the effective 4-dimensional cosmological constant, $\bar{\Lambda}_{4}$, is given in terms of its 5 -dimensional analogue, $\Lambda$, and the force, $\bar{f}$, by

$$
\frac{\bar{\Lambda}_{4}}{3}=\frac{\Lambda}{6}+\left(\frac{\bar{f}}{4 \mathcal{T}_{\infty}}\right)^{2}
$$

It can immediately be verified that (80) agrees with what is obtained from the corresponding formula in the preceding analysis [3] when the background gravitational coupling constant is set to zero. However, as we have already pointed out, if we similarly set the background gravitational coupling to zero in the corresponding formula [3] for the effective 4-dimensional gravitational coupling constant $G_{[4]}$ we would obtain a negative value that cannot possibly matched to the Newton constant $\mathrm{G}_{\mathrm{N}}$ that is actually observed. This is because the scheme of the previous work corresponds to assuming that the constant $E_{\mathrm{B}}$ is sufficiently small for its contribution to be entirely neglected in the late time limit considered here, so that the bracketed contribution in (79) contains at lowest order just the $\varepsilon$ term, which has the desired property of proportionality to $\rho$, but with the wrong sign.

The idea of the present approach is to get round this apparent obstacle to taking the zero background gravity limit by making an essentially different hypothesis, namely that instead of being small, $E_{\mathrm{B}}$ is large enough to give a positive contribution that can overcome the negative contribution proportional to $-\varepsilon$. The cosmological scenario will thus have an era dominated by what we refer to as pseudo-radiation, that will last until at least the present epoch the unless it was superseded by a transition (which if it occurred must have been recent) to an epoch of 
domination by a cosmological constant. Instead of the term "mirage matter" that was used by Kehagias and Kiritsis [5] whose pioneering analysis of gravitationally uncoupled brane-world scenarios originally drew attention to the potential cosmological importance of such an effect, we prefer to use the term "pseudo-radiation" for the background geometric contribution dominating the effective Friedmann equation because, like a true radiation gas, its energy density evolves proportionally to $a^{-4}$, while unlike an ordinary mirage its dynamical effect is very real - in our case actually preponderant.

\subsection{The nucleosynthesis condition}

In order for the kind of scenario described in the preceding subsection to be realistic, we need at least to require that the four parameters $\left(\Lambda, \mathcal{T}_{\infty}, \bar{f}, E_{\mathrm{B}}\right)$ are such that at nucleosynthesis, the pseudo-radiation term dominates the Friedmann equation (in particular compared with terms in $a^{-8}$ ) and that its amplitude has the value required to get the correct nuclear abundances, i.e. when the temperature, $\Theta$, is comparable with the nuclear reaction value, $\Theta_{\mathrm{N}} \approx 1 \mathrm{MeV}$, the expansion rate must be given approximately by

$$
\left(\frac{\dot{a}}{a}\right)^{2} \simeq \frac{8 \pi \mathrm{G}_{\mathrm{N}}}{3} \rho_{\mathrm{r}}
$$

where $\rho_{\mathrm{r}}$ is the radiation density as given by the formula

$$
\rho_{\mathrm{r}}=\frac{\pi^{2}}{30} g_{*} \Theta^{4},
$$

in which the value of the number $g_{*}$ of effective relativistic degrees of freedom is calculated to allow for the photon field and three families of degenerate neutrinos which gives $g_{*}=10.75$. In order to avoid getting a negative value for the right hand side of (79) at some subsequent time, the parameters must be such that the pseudo radiation contribution proportional to $a^{-4}$, or equivalently to $\Theta^{4}$, must be larger than the true radiation contribution given by (82) and also than the matter contribution $\rho_{\mathrm{m}}$ in the decomposition $\rho=\rho_{\mathrm{m}}+\rho_{\mathrm{r}}$ In order to be consistent with the standard picture of development of clustering from small perturbations the cosmological constant must remain subdominant until at least the present epoch.

To determine the implication from these requirements, we expand (79) to get

$$
\left(\frac{\dot{a}}{a}\right)^{2}=\frac{\bar{\Lambda}_{4}}{3}+\frac{1}{\mathcal{T}_{\infty}^{2}}\left[\frac{\bar{f}}{2} \frac{E_{\mathrm{B}}}{a^{4}}+\frac{E_{\mathrm{B}}^{2}}{a^{8}}-2 \varepsilon\left(\frac{\bar{f}}{4}+\frac{E_{\mathrm{B}}}{a^{4}}\right)^{2}\right]+\mathcal{O}\left(\varepsilon^{2}\right)
$$

and make the decomposition $\varepsilon=\varepsilon_{\mathrm{m}}+\varepsilon_{\mathrm{r}}$, where $\varepsilon_{\mathrm{m}}=\rho_{\mathrm{m}} / \mathcal{T}_{\infty}$ and $\varepsilon_{\mathrm{r}}=\rho_{\mathrm{r}} / \mathcal{T}_{\infty}$. These terms will be given in terms of their present values by $\varepsilon_{\mathrm{m}}=\varepsilon_{\mathrm{m}}^{0} \vartheta^{3}$ and $\varepsilon_{\mathrm{r}}=\varepsilon_{\mathrm{r}}^{0} \vartheta^{4}$, in terms of the reduced temperature, as defined in terms of its present value $\Theta_{0}$ or the present value $a_{0}$ of the cosmological scale factor by

$$
\vartheta=\frac{\Theta}{\Theta_{0}}=\frac{a_{0}}{a} .
$$

The equation (83) is thereby expressible in the form, after setting $a_{0}=1$,

$$
\begin{gathered}
\left(\frac{\dot{a}}{a}\right)^{2}=\frac{\bar{\Lambda}_{4}}{3}+\frac{1}{\mathcal{T}_{\infty}^{2}}\left[\frac{\bar{f}}{2}\left(E_{\mathrm{B}}-\frac{\bar{f}}{4} \varepsilon_{\mathrm{r}}^{0}\left(1+\frac{\vartheta_{\text {eq }}}{\vartheta}\right)\right) \vartheta^{4}+E_{\mathrm{B}}\left(E_{\mathrm{B}}-\bar{f}_{\varepsilon_{\mathrm{r}}^{0}}\left(1+\frac{\vartheta_{\text {eq }}}{\vartheta}\right)\right) \vartheta^{8}\right. \\
\left.-2 E_{\mathrm{B}}^{2} \varepsilon_{\mathrm{r}}^{0}\left(1+\frac{\vartheta_{\text {eq }}}{\vartheta}\right) \vartheta^{12}\right]+\mathcal{O}\left(\varepsilon^{2}\right)
\end{gathered}
$$


where we have introduced the matter-radiation density equality value

$$
\vartheta_{\text {eq }} \equiv \frac{\varepsilon_{\mathrm{m}}^{0}}{\varepsilon_{\mathrm{r}}^{0}}
$$

of the reduced temperature, which is much larger than unity $\left(\vartheta_{\text {eq }} \sim 10^{3}\right.$ in standard cosmology $)$ and in terms of which the total matter component takes the form

$$
\varepsilon=\varepsilon_{\mathrm{r}}^{0} \vartheta^{3}\left(\vartheta+\vartheta_{\text {eq }}\right) .
$$

Note that the terms of $\mathcal{O}\left(\varepsilon^{2}\right)$ in (85), although containing terms of order $\vartheta^{6}$, are suppressed by an additional factor of $\varepsilon_{\mathrm{r}}^{0}$ and thus can be dropped legitimately (see below).

In order for the term proportional to $\vartheta^{4}$ to remain positive until at least the present time, $\vartheta \simeq 1$, we evidently need that the pressureless matter term be smaller that the pseudo radiation term proportional to $E_{\mathrm{B}}$, giving the constraint

$$
4 E_{\mathrm{B}}>\bar{f} \varepsilon_{\mathrm{r}}^{0} \vartheta_{\text {eq }}
$$

and furthermore, to avoid premature domination by the cosmological constant, we also need

$$
\bar{f}\left(4 E_{\mathrm{B}}-\bar{f} \varepsilon_{\mathrm{r}}^{0} \vartheta_{\text {eq }}\right) \geq \frac{8}{3}\left|\Lambda_{4}\right| \mathcal{T}_{\infty}^{2} .
$$

This implies that the term in $\vartheta^{3}$, which has the wrong sign, must always have been subdominant until now: it can at most equal the pseudo-radiation component today, and thus was much less in the past since it redshifts more slowly.

Since the true radiation term is always a fixed fraction of the pseudo-radiation term, the fact that our scenario is compatible with the nucleosynthesis data leads first to the identification of the Newton constant, $\mathrm{G}_{\mathrm{N}}$ in the formula (81) as

$$
\mathrm{G}_{\mathrm{N}}=\frac{3 \bar{f}}{16 \pi \mathcal{T}_{\infty}^{3}}\left(\frac{E_{\mathrm{B}}}{\varepsilon_{\mathrm{r}}^{0}}-\frac{\bar{f}}{4}\right) .
$$

Since $\vartheta_{\text {eq }} \gg 1$, the positivity of $\mathrm{G}_{\mathrm{N}}$ is evidently ensured by (88). It is to be observed that this globally determined quantity $\mathrm{G}_{\mathrm{N}}$ is a priori different from the quantity $\mathrm{G}_{[4]}$ determined according to (62) by local physics, so the requirement that they should ultimately agree will entail a rather excessive amount of fine tuning.

In order for the term in (85) proportional to $\vartheta^{8}$ to be negligible compared to the one proportional to $\vartheta^{4}$ at the epoch of nucleosynthesis we evidently need to have

$$
\bar{f}\left(4 E_{\mathrm{B}}-\bar{f} \varepsilon_{\mathrm{r}}^{0}\right)>8 E_{\mathrm{B}}\left(E_{\mathrm{B}}-\bar{f} \varepsilon_{\mathrm{r}}^{0}\right) \vartheta_{\mathrm{N}}^{4}
$$

where $\vartheta_{\mathrm{N}} \sim 10^{10}$ is the value of $\vartheta$ at nucleosynthesis. In conjunction with (88), this latter constraint simply gives the requirement that the four parameters $E_{\mathrm{B}}, \bar{f}, \mathcal{T}_{\infty}$ and $\Lambda$ should satisfy the conditions expressible in terms of the present cosmological radiation density

$$
\rho_{\mathrm{r}}^{0}=\mathcal{T}_{\infty} \varepsilon_{\mathrm{r}}^{0}=\frac{\pi^{2}}{30} g_{*} \Theta_{0}^{4}
$$


as the inequalities

$$
\vartheta_{\mathrm{N}}^{-4} \gg 2 \frac{E_{\mathrm{B}}}{\bar{f}}>\frac{\rho_{\mathrm{r}}^{0} \vartheta_{\mathrm{eq}}}{\mathcal{T}_{\infty}}
$$

The only other restrictions on the four listed parameters are the conditions obtained from the inequality (89) and the identification (90) — in which the second term on the right is negligible by (88) - so that we are left with

$$
\frac{16 \pi \rho_{\mathrm{r}}^{0}}{3 m_{\mathrm{P}}^{2}} \simeq \frac{\bar{f} E_{\mathrm{B}}}{\mathcal{T}_{\infty}^{2}} \gtrsim \frac{2}{3} \bar{\Lambda}_{4},
$$

using the standard definition, $m_{\mathrm{P}} \equiv \mathrm{G}_{\mathrm{N}}^{-1 / 2}$, of the Planck mass, whose numerical value is given by $m_{\mathrm{P}} \approx 10^{22} \mathrm{MeV}$. No further restriction is needed to ensure that the term proportional to $\vartheta^{12}$ in (83) remains negligible at temperatures up to the nucleosynthesis range.

It can now be seen to follow directly from (93) that the brane mass scale $m_{\infty}$, defined by $m_{\infty}^{4} \equiv \mathcal{T}_{\infty}$, must satisfy

$$
m_{\infty} \gg\left(\rho_{\mathrm{r}}^{0} \vartheta_{\text {eq }}\right)^{1 / 4} \vartheta_{\mathrm{N}} \text {. }
$$

With the standard values $\vartheta_{\text {eq }} \approx 10^{3}, \vartheta_{\mathrm{N}} \approx 10^{10}$ and $\Theta_{0} \approx 2 \times 10^{-10} \mathrm{MeV}$, which corresponds to $\rho_{\mathrm{r}}^{0} \sim 6 \times 10^{-39}(\mathrm{MeV})^{4}$, we get that

$$
m_{\infty} \gg 10 \mathrm{MeV}
$$

which is comparable with what we found in our previous work [3]. The bound (96) implies that $\varepsilon_{\mathrm{r}}^{0} \ll 10^{-44}$ which justify a posteriori that we can neglect terms of order $\varepsilon^{2}$ in (85).

The first equality in (94) will now give an order of magnitude inequality for the product $\bar{f} E_{\mathrm{B}}$, which will be given in terms of the brane tension by

$$
\frac{\bar{f} E_{\mathrm{B}}}{\mathcal{T}_{\infty}^{2}} \approx 10^{-81}(\mathrm{MeV})^{2}
$$

and hence by $(96)$ will satisfy $\bar{f} E_{\mathrm{B}} \gtrsim 10^{-73}(\mathrm{MeV})^{10}$. In order for the cosmological constant to have become important by the present epoch (as suggested by some recent supernova data) we would need that the ratio $\bar{\Lambda}_{4} / H_{0}^{2}$ should be comparable with unity where $H_{0}$ is the present day value of the Hubble expansion rate which is given roughly by where $H_{0} \approx 10^{-38} \mathrm{MeV}$. However it follows from (94) that this condition would fail by a considerable margin since substitution of (97) gives

$$
\bar{\Lambda}_{4} / H_{0}^{2} \lesssim 10^{-11}
$$

The limits on $E_{\mathrm{B}}$ obtained from (93) by substitution from the first equality in (94) have the form

$$
10^{-56}\left(\frac{m_{\infty}}{10 \mathrm{MeV}}\right)^{4} \mathrm{MeV}^{5} \gg E_{\mathrm{B}}>10^{-56}\left(\frac{m_{\infty}}{10 \mathrm{MeV}}\right)^{2} \mathrm{MeV}^{5}
$$

\section{Discussion}

It seems from the former paragraph that we can find a set of parameters for our model to be compatible with large-scale cosmology (that is, with nucleosynthesis and a cosmological constant 
dominated universe today), even if very contrived. But it has to be emphasized that the largescale value of the gravitational constant

$$
\mathrm{G}_{\mathrm{N}}=\frac{3}{8 \pi \mathcal{T}_{\infty}^{3}} \frac{\bar{f}}{2}\left(\frac{E_{\mathrm{B}}}{\varepsilon_{\mathrm{r}}^{0}}-\frac{\bar{f}}{4}\right)
$$

has no reason a priori to agree with its small scale value (62)

$$
\mathrm{G}_{[4]}=\frac{\left(K_{00}^{(\text {local })}\right)^{2}}{4 \pi \mathcal{T}_{\infty}}
$$

We first investigate here, the compatibility between the local and global properties of the gravitation and then discuss the viability of such a model as well as its possible extensions.

\subsection{Compatibility between local and global matching}

To compare the two values of the Newton constant, respectively determined from the local Newton law and global cosmology, we need to compute the extrinsic curvature in the cosmological framework. From (38) we get, at first order in $\varepsilon$, that the extrinsic curvature scalar is given by

$$
K^{\text {(global) }}=-\frac{\bar{f}}{\mathcal{T}_{\infty}}+\left[\frac{6+\Gamma}{4} \bar{f}+3 \Gamma E_{\mathrm{B}} \vartheta^{4}\right] \frac{\varepsilon}{\mathcal{T}_{\infty}}+\mathcal{O}\left(\varepsilon^{2}\right)
$$

on the assumption that, as described in more detail in the appendix, one assumes a polytropic equation of state with index $\Gamma$ for which the pressure of the cosmic fluid in (41) will be given by $P=(\Gamma-1) \rho$. Equation (28) then implies that we shall have

$$
K_{00}^{\text {(global) }}=\frac{\bar{f}}{4 \mathcal{T}_{\infty}}-\frac{3}{\mathcal{T}_{\infty}} E_{\mathrm{B}} \vartheta^{4}-\left[\bar{f}-3(\Gamma+1)\left(\frac{\bar{f}}{4}+E_{\mathrm{B}} \vartheta^{4}\right)\right] \frac{\varepsilon}{\mathcal{T}_{\infty}}+\mathcal{O}\left(\varepsilon^{2}\right) .
$$

In the relevant range (93) for the admissible parammeter values, the large-scale value of the extrinsic curvature components will therefore be given at lowest order in $\varepsilon$ today (i.e. when $\vartheta \sim 1$ ) by

$$
K_{\text {(global) }} \simeq-\frac{\bar{f}}{\mathcal{T}_{\infty}}, \quad K_{\text {oo }}^{\text {(global) }} \simeq-\frac{1}{\mathcal{T}_{\infty}} \frac{\bar{f}}{4}
$$

The extrinsic curvature vector will thus be subject to what is interpretable as a space-time isotropy (Lorentz invariance) condition, namely

$$
K_{00}^{\text {(global) }}=-\frac{1}{4} K^{\text {(global) }}
$$

which is evidently quite different from the observational constraint (66). However the apparent contradiction between these two conditions need not be of any consequence if, as we have already concluded, the relevant local and global curvature values are independent.

To reconcile the cosmologically determined value $\mathrm{G}_{\mathrm{N}}$ given by (100) with the locally measured value $\mathrm{G}_{[4]}$, given by (101), of the gravitational constant we need to have

$$
K_{00}^{(\text {local })} \simeq \pm\left(\frac{3 \bar{f}}{4 \mathcal{T}_{\infty}^{2}} \frac{E_{\mathrm{B}}}{\varepsilon_{\mathrm{r}}^{0}}\right)^{1 / 2}
$$


with the supplementary requirement $K^{(\text {local })}=2 K_{00}^{(\text {local })}$. This local value is obviously different from the value (104) found on large scales since we shall have

$$
\left|K_{00}^{\text {(global) }}\right|=\alpha\left|K_{00}^{\text {(local) }}\right| \quad \text { with } \quad \alpha^{2} \equiv 12 \varepsilon_{\mathrm{r}}^{0} \frac{\bar{f}}{E_{\mathrm{B}}},
$$

where by (93), we get that the parameter $\alpha$ is in the range

$$
2 \times 10^{-3} \gtrsim \alpha^{2} \gtrsim 9 \times 10^{-3}\left(\frac{m_{\infty}}{10 \mathrm{Mev}}\right)^{-4}
$$

from which it follows, a fortiorei, that $\alpha<1$.

Such mismatch between the small-scale and large-scale values of the extrinsic curvature does not seem unreasonable in view the consideration that the background configuration of brane does not need to be strictly homogeneous and isotropic, as assumed until now, but may have different curvature on small and large scales. The cosmological constraints determine the largescale extrinsic curvature, while light deflection observations so far give precise information only about local extrinsic curvature. This raises the question of the transition scale between these two regimes which should range somehow above the galactic scale. This also leads to a potentially lethal test for our model: since the local extrinsic curvature has no reason to be the same in two different galaxies, the measurement of the light deflection should lead to different results in different galaxies.

\subsection{Viability of the model}

We have shown that as well as automatically providing a local gravitational interaction of the familiar Newtonian kind, the simulated gravity model presented here can be contrived in such a way as to satisfy the most stringent cosmological constraint - namely the one provided by nucleosynthesis. However it is still confronted with other serious problems.

- Firstly, it suffers from a double fine tuning requirement arising from the fact that, on local scales, the gravitational constant depends a priori on time. We must require not only that the weak-field limit be instantaneously compatible with the the condition (66) for consistency with the observed deflection of light, but also that the predicted gravitational constant (62) should vary slowly enough to be consistent with the experimental limit [23]

$$
\left|\frac{\dot{\mathrm{G}}_{[4]}}{\mathrm{G}_{[4]}}\right| \leq 6 \times 10^{-12} \mathrm{yr}^{-1} .
$$

- Secondly, the predicted post-Newtonian effects and the current observational limits [17] imply that the local value extrinsic curvature is subject not just to the limitation its mixed components $K_{0} a$ given by (67) and (68) but on the further fine tuning condition subjecting its time component $K_{00}$ to (66), which is eqivalent to the condition that the extrinsic curvature should turn out to be almost exactly of the form

$$
K_{\mu \nu}=K_{00}\left(2 \bar{u}_{\mu} \bar{u}_{\nu}+\bar{\gamma}_{\mu \nu}\right),
$$

entailing $\nu^{\prime} \sim-r^{\prime} / a$ in (28). Note that, at this stage of our investigation, these restrictions are not worse than those occuring e.g. for scalar-tensor theories of gravity (see [19] for 
a comparison) before the mechanism of attraction [21] toward General Relativity was discovered. However at this stage we are not aware of any natural mechanism that would lead to the form (110) satisfying the post-Newtonian constraints as a preferred outcome.

In addition to the kinds of observational restriction we have considered so far, it is to be noted that there will be others involving non-linear effects and motion of the source. It is not our purpose to investigate all these constraints here, but just to point out that they are of potential importance whenever alternative theories of gravity are under consideration, and that merely recovering the standard Newton law is in general not enough. None of these post-Newtonian constraints have been explicitly taken into account so far in this or other works on brane-world scenarios. However, in typical brane-world models, (even those of asymmetric type) gravity at Solar system scales is describable in terms of a spin 2 graviton field, and in such cases it can be expected that the post-Newtonian constraint will automatically be satisfied.

- Thirdly, concerning the cosmology of our model, the expansion rate switches from a pseudoradiation to a cosmological constant dominated phase whereas the matter content switches from a radiation dominated era to a matter dominated era (at $\vartheta_{\text {eq }}$ ) and then to a cosmological constant dominated era (at $\vartheta \sim 1$ ). This is a non-standard cosmological scenario that does not have an ordinary matter-dominated era characterised by a Friedmann type evolution equation of the form $H^{2} \propto a^{3}$. raises the question of how large-scale structure will develop in this kind of scenario. In the case of the standard cosmology, it is known that density perturbations needed for subsequent galaxy formation cannot grow during a radiation dominated era. However it is not yet clear what may happen in a pseudo radiation era in which the strength of gravity on shorter scales is affected by the nature of fluctuations on longer scales.

- Fourthly, in relation to the preceeding remark, we need a transition scale between the local and global behaviour that has to range a somewhere above the galaxy scale. However, this scale is not directly determined by the model and might involve a third fine tuning. A potentially relevant test that can be thought of is weak gravitational lensing on cosmological scales 22] which can provide a probe of the Newtonian law of gravitation (and more particularly of the Poisson equation) up to some hundreds of Megaparsec when data are available. This may be able to provide constraints on this transition scale.

\subsection{Conclusions}

In this article, we have considered a simulated gravity model when the reflection symmetry is broken in brane-world scenarios for which genuine (bulk) gravitational coupling is absent, but in which the possible effect of a gauge four form coupling is allowed for. A previous investigation by Kehagias and Kiritsis [5] of this gravitationally decoupled "probe" limit? is noteworthy for having drawn attention to the dynamical importance of what they referred to as "mirage" matter, meaning the contribution we have preferred to describe as pseudo-radiation. Their work was restricted to homogeneous isotropic cosmological configurations of any codimension, and allowed only for the kinds of matter field most directly motivated by higher dimensional superstring theory, namely a Born-Infeld type contribution in the brane and a Ramond-Ramond

\footnotetext{
${ }^{2}$ The motion of these they sometimes describe as "geodesic motion of a probe D3-brane", which is potentially misleading as the evolution is not generated by geodesics in the usual sense of the term.
} 
gauge field outside. In this investigation we restrict attention to the codimension one case but progress further in two ways. The first advance is by allowing for more realistic matter fields on the brane, and showing that the so-called "mirage" contribution, which we have called pseudoradiation, can be adjusted to give consistency with nucleosynthesis - the most empirically sensitive cosmological constraint. The second kind of progress made here is the less mathematically trivial step of allowing for local deviations from homogeneity and the demonstration that in the weak-field, small-scale limit there will be an effective "simulated" gravity mechanism with a Newtonian type inverse square law behaviour.

The outcome of the first advance is rather satisfactory: having derived the equation of evolution of the universe we show that, for a certain range of parameters, it can be made compatible with nucleosynthesis subject to the proviso that the cosmological constant is not yet dominant today. However the outcome of the second kind of progress raises as many problems as it solves: as discussed in the previous section, agreement at the local level can only be obtained by adjusting the relevant parameters in an artificially contrived manner. It necessitates at least two fine tunings (arising from the time variation of the gravitational constant and of the compatibility with the post-Newtonian limit), and requires an undetermined transition lengthscale between local and global properties of our 3-brane. Let us emphasize that it is not enough to recover Newton gravity on local scales and to produce a viable cosmology; one also needs to take the post-Newtonian constraints into account. Further work would be needed to derive the detailed evolution of density fluctuations in our model, but it does not seem that it would lead to an acceptable description of structure formation, at least if the standard picture is still valid (which is not entirely obvious).

Different, more sophisticated, scenarios might be conceived, in which one might try to overcome the problems that have been pointed out by allowing more general fields to act in the bulk. Another possibility is to change the background geometry, which we can do without changing the bulk matter content when there is no gravity. By tuning the background, it is very likely that we could get a cosmology with the standard Friedmann law. In any case, even if the effective gravity mechanism described here is not dominant, it will always be present to some extent as a contributing effect in reflection symmetry breaking brane-world scenarios. Whereas most previous work has concentrated on strongly self gravitating reflection symmetric case, the present article initiates investigation of the opposite extreme limiting case of brane-worlds that are very weakly self gravitating but for which gravity is simulated by the effect of reflection symmetry violation.

\section{Acknowledgements}

We wish to thank Daniele Steer for interesting discussions on moving branes, and Gilles EspositoFarèse for discussions on scalar-tensor theories. We have also benefitted from informative discussions with José Blanco-Pillado and Martin Bucher about previous relevant work [16] on 2-branes. We are grateful for support from P.P.A.R.C. (for R.A.B.) and from E.P.S.R.C. (for A.M.).

\section{A Variational formulation in static background}

The variational principle for a minimally coupled 3-brane in a given background specified by a metric $g_{\mu \nu}$ and a (Ramond-Ramond type [5]) gauge 4 form $A_{\mu \nu \rho \sigma}$ will be given [4] in terms 
of a Lagrangian density $\mathcal{L}$ depending only on internal fields on the brane worldsheet, and of a "brawn" coupling (generalised charge) constant $e_{[4]}$, by an action integral of the form

$$
\mathcal{I}=\int\left(\mathcal{L}+\frac{e_{[4]}}{4 !} \mathcal{E}^{\mu \nu \rho \sigma} A_{\mu \nu \rho \sigma}\right) \mathrm{d} \overline{\mathcal{S}}
$$

where $\mathrm{d} \overline{\mathcal{S}}$ is the element of worldsheet volume measure as given in terms of internal coordinates $\sigma^{i},(i=0,1,2,3)$ and the determinant $|\bar{\gamma}|$ of the induced metric $\bar{\gamma}_{i j}$ by

$$
\mathrm{d} \overline{\mathcal{S}}=\|\bar{\gamma}\|^{1 / 2} \mathrm{~d} \sigma^{0} \mathrm{~d} \sigma^{1} \mathrm{~d} \sigma^{2} \mathrm{~d} \sigma^{3},
$$

and where $\mathcal{E}^{\mu \nu \rho \sigma}$ is the corresponding antisymmetric unit worldsheet tangent tensor (normalised so that $\mathcal{E}^{\mu \nu \rho \sigma} \mathcal{E}_{\mu \nu \rho \sigma}=4$ !) as specified in terms of the associated internal measure tensor $\mathcal{E}_{\text {hijk }}$ by

$$
\mathcal{E}^{\mu \nu \rho \sigma}=\mathcal{E}^{h i j k} x_{, h}^{\mu} x_{, i}^{\nu} x_{, j}^{\rho} x_{, k}^{\sigma} .
$$

For a bulk metric of the static form (23) the space metric $\mathrm{d} \hat{s}^{2}=r^{3} \mathrm{~d} \ell^{2}$ of the homogeneous isotropic space sections will similarly determine an analogous antisymmetric tensor $\hat{\mathcal{E}}^{\mu \nu \rho}$ that is tangent to the homogeneous isotropic space 3 -sections with normalisation $\hat{\mathcal{E}}^{\mu \nu \rho} \hat{\mathcal{E}}_{\mu \nu \rho}=6$. This space (as opposed to space time) measure tensor can be used to define a conformally related tensor

$$
\mathcal{Y}_{\mu \nu \rho}=r \hat{\mathcal{E}}_{\mu \nu \rho}
$$

with the noteworthy property of having a covariant derivative that is entirely antisymmetric, which qualifies it for description as a Killing Yano tensor. This special property can be seen to follow just from the "warped product" form (23) of the full spacetime metric $\mathrm{d} s^{2}$ (independently of the special homogeneity and isotropy property postulated for $\mathrm{d} \ell^{2}$ ) which enables us to evaluate the covariant derivative as

$$
\nabla_{\mu} \mathcal{Y}_{\nu \rho \sigma}=\frac{4}{r} r_{[, \mu} \mathcal{Y}_{\nu \rho \sigma]}
$$

It follows that the symmetric product tensor

$$
\mathcal{K}_{\mu \nu}=\frac{1}{2} \mathcal{Y}_{\mu \rho \sigma} \mathcal{Y}_{\nu}{ }^{\rho \sigma}
$$

with trace $\mathcal{K}_{\mu}{ }^{\mu}=3 r^{2}$, will be an ordinary Killing tensor, meaning that its symmetrised derivative vanishes,

$$
\nabla_{(\mu} \mathcal{K}_{\nu \rho)}=0
$$

so that for a free particle motion it determines a quadratic constant of motion of the kind recently discussed in the general context of "warped product" metrics by Droz-Vincent [24].

The utility of the Killing Yano tensor $\mathcal{Y}_{\mu \nu \rho}$ for our present purpose is that it can be used to construct an explicit static solution for the "brawn" gauge field $A_{\mu \nu \rho \sigma}$, which may be taken to be given in terms of the gradient of the time coordinate $t$ in the expression (23) for the static bulk metric by

$$
A_{\mu \nu \rho \sigma}=F t_{[, \mu} \mathcal{Y}_{\nu \rho \sigma]},
$$

where $F$ is a constant, which will determine the value of the force density $\bar{f}$ on the right hand side of the equation of motion (20) according to the prescription [ [ ]

$$
\bar{f}=e_{[4]} F,
$$


and in terms of which the corresponding gauge independent field tensor,

$$
F_{\mu \nu \rho \sigma \tau}=5 \nabla_{[\mu} A_{\nu \rho \sigma \tau]}
$$

can be seen to be given simply by

$$
F_{\mu \nu \rho \sigma \tau}=F \epsilon_{\mu \nu \rho \sigma \tau}
$$

where $\epsilon_{\mu \nu \rho \sigma \tau}$ is the background spacetime measure tensor, which will be expressible in terms of the coordinates introduced in (23) by

$$
\epsilon_{\mu \nu \rho \sigma \tau}=20 r_{,[\mu} t_{, \nu} \hat{\mathcal{E}}_{\rho \sigma \tau]} .
$$

Let us now specialise, as in $\S$ 3, to the case in which the brane is itself homogeneous and isotropic, so that the evolution of its worldsheet will be describable simply by specifying $r$ as a function of $t$. In this case the worldsheet tangent tensor $\mathcal{E}^{\mu \nu \rho \sigma}$ will simply be specifiable in terms of the preferred cosmological unit vector $\bar{u}^{\mu}$ introduced in (28) by

$$
\mathcal{E}^{\mu \nu \rho \sigma}=4 \bar{u}^{[\mu} \hat{\mathcal{E}}^{\nu \rho \sigma]},
$$

and hence by (118) the coupling term in (111) will be given just by

$$
\frac{e_{[4]}}{4 !} \mathcal{E}^{\mu \nu \rho \sigma} A_{\mu \nu \rho \sigma}=\frac{e_{[4]} F}{4} \bar{u}^{\mu} t_{, \mu} .
$$

In the homogeneous isotropic case (and even more generally for the treatment of adiabatic cosmological perturbations) little generality will be lost by supposing that the matter on the brane is describable as an irrotational perfect fluid characterised by an equation of state specifying the energy density $\bar{\rho}$ as a function of the number density, $\bar{n}$ say of conserved particles, which (in the subnuclear energy regime we are concerned with) can taken to be baryons. In such a case the 4-dimensional fluid dynamics will have a particularly simple Lagrangian formulation in which the only independent variable is a scalar potential, $\varphi$ say, with a timelike gradient $\varphi, \rho$ whose magnitude $\bar{\mu}$, as given by

$$
\bar{\mu}^{2}=-\bar{\gamma}^{i j} \varphi_{, i} \varphi, j
$$

is to be identified with the relevant chemical potential (i.e. effective mass per baryon) as defined by

$$
\bar{\mu}=\frac{\mathrm{d} \bar{\rho}}{\mathrm{d} \bar{n}},
$$

while the Lagrangian itself can be taken to be the corresponding pressure function $\bar{P}$, as obtained in terms of $\bar{\mu}$ by application of the standard prescription

$$
\bar{P}=\bar{n} \bar{\mu}-\bar{\rho} .
$$

To adapt this simple perfect fluid model to the context of a brane-world in a 5-dimensional background, all one has to do is to identify the Lagrangian density $\mathcal{L}$ in (111) with the negative of the total brane tension $\mathcal{T}$ as given by (41), i.e. we take

$$
\mathcal{L}=-\mathcal{T}
$$


with

$$
\mathcal{T}=\mathcal{T}_{\infty}-\bar{P}
$$

In order to be explicit, for typical cosmological purposes a good description will be provided by an equation of state of polytropic form given in terms of a fixed mass (per baryon) $m$, a fixed polytropic index $\Gamma$, and a fixed proportionality constant $\kappa$ say by

$$
\bar{\rho}=\bar{\rho}_{\mathrm{m}}+\bar{\rho}_{\mathrm{r}},
$$

with

$$
\bar{\rho}_{\mathrm{m}}=m \bar{n}, \quad \rho_{\mathrm{r}}=\kappa \bar{n}^{\Gamma},
$$

which gives

$$
\bar{\mu}=m+\kappa \Gamma \bar{n}^{\Gamma-1}, \quad \bar{P}=(\Gamma-1)(\bar{\rho}-m \bar{n}),
$$

so that the required pressure function is finally obtained in the form

$$
\bar{P}=\kappa(\Gamma-1)\left(\frac{\bar{\mu}-m}{\kappa \Gamma}\right)^{\Gamma /(\Gamma-1)} .
$$

The simplest relevant possibility is that of a pure radiation gas, as characterised by $m=0$ and $\Gamma=4 / 3$ for which the pressure function contribution to the Lagrangian density will just be proportional to $\left(\bar{\gamma}^{i j} \varphi_{, i} \varphi, j\right)^{2}$.

Whatever the form of the equation of state, the four dimensional surface integral in (111) will be proportional in the homogeneous isotropic case to a one dimensional integral of the form

$$
I=\int r^{3}\left(-\mathcal{T} \mathrm{d} \tau+e_{[4]} F \frac{r}{4} \mathrm{~d} t\right) .
$$

Using the explicit form of the metric (23) to evaluate the derivative of the proper time $\tau$ with respect to the background metric time $t$, this will be expressible in the standard form

$$
I=\int L \mathrm{~d} t
$$

for an ordinary Lagrangian (not Lagrangian density) function given by

$$
L=-\mathcal{T}\left(\mathcal{V}-\mathcal{V}^{-1}\left(\frac{\mathrm{d} r}{\mathrm{~d} t}\right)^{2}\right)^{1 / 2} r^{3}+e_{[4]} F \frac{r^{4}}{4}
$$

in which the equation of state specifies $\bar{P}$, and hence also $\mathcal{T}$, as a function of $\bar{\mu}$ and hence of $\mathrm{d} \varphi / \mathrm{d} t$ and $\mathrm{d} r / \mathrm{d} t$ via the relation

$$
\bar{\mu}=\frac{\mathrm{d} \varphi}{\mathrm{d} \tau}=\frac{\mathrm{d} \varphi}{\mathrm{d} t}\left(\mathcal{V}-\mathcal{V}^{-1}\left(\frac{\mathrm{d} r}{\mathrm{~d} t}\right)^{2}\right)^{-1 / 2} \mathcal{V}^{-1 / 2} .
$$

In terms of the momenta defined by the variation

$$
\mathrm{d} L=\frac{\partial L}{\partial r} \mathrm{~d} r+p_{r} \mathrm{~d}\left(\frac{\mathrm{d} r}{\mathrm{~d} t}\right)+p_{\varphi} \mathrm{d}\left(\frac{\mathrm{d} \varphi}{\mathrm{d} t}\right),
$$

the associated Hamiltonian function will be given by an expression of the standard form

$$
H=p_{\varphi} \frac{\mathrm{d} \varphi}{\mathrm{d} t}+p_{r} \frac{\mathrm{d} r}{\mathrm{~d} t}-L .
$$


It is to be remarked that this ordinary kind of Hamiltonian, as defined with respect to the background time coordinate $t$, is to be distinguished from the alternative kind of Hamiltonian defined with respect to the proper time $\tau$ (as used in the Wheeler De Witt type formulation [7]), whose use requires the imposition of an extra constraint (whereby the proper time Hamiltonian is initially set to zero in the standard version of the Wheeler De Witt formulation). In the present case the relevant momenta are found to be given by the formulae

$$
p_{\varphi}=\bar{n} r^{3}, \quad p_{r}=\left(\mathcal{V}-\mathcal{V}^{-1}\left(\frac{\mathrm{d} r}{\mathrm{~d} t}\right)^{2}\right)^{-1 / 2} \mathcal{V}^{-1 / 2} \mathcal{U} r^{3} \frac{\mathrm{d} r}{\mathrm{~d} t}
$$

in which $\mathcal{U}$ is the energy density function given according to (41) by

$$
\mathcal{U}=\mathcal{T}_{\infty}
$$

We thus obtain the Hamiltonian in the form

$$
H=\left(\mathcal{V}-\mathcal{V}^{-1}\left(\frac{\mathrm{d} r}{\mathrm{~d} t}\right)^{2}\right)^{-1 / 2} \mathcal{V} \mathcal{U} r^{3}+e_{[4]} F \frac{r^{4}}{4}
$$

The time independence of the background ensures that the motion will be such that this Hamiltonian has a constant value

$$
H=E_{\mathrm{B}},
$$

that is identifiable as the integration constant introduced in (36), which is thus interpretable as a cosmological energy density. A more trivial observation is that since the Lagrangian does not depend on the undifferentiated scalar $\varphi$ but only on its gradient (i.e. since (138) contains no $\partial L / \partial \varphi$ term) the corresponding momentum $p_{\varphi}$ will also be conserved, a conclusion that can be seen from (140) to be interpretable as equivalent to conservation of baryons, whose number density varies inversely as the cosmological volume, i.e. $\bar{n} \propto r^{-3}$.

As a final remark concerning energy conservation, it is to be observed that the stationary character of the background specified by (23) and (118) with respect to the action generated by the Killing vector $k^{\mu}$ given by $k_{\mu} \mathrm{d} x^{\mu}=-\mathcal{V} \mathrm{d} t$ ensures that even when inhomogeneous perturbations are taken into account there will still be an energy density current, $\bar{E}^{\mu}$ say, given by

$$
\bar{E}^{\mu}=k^{\lambda}\left(\bar{T}_{\lambda}^{\mu}+\frac{e_{[4]}}{4 !} A_{\lambda \nu \rho \sigma} \mathcal{E}^{\mu \nu \rho \sigma}\right)
$$

that will satisfy a surface flux conservation law of the simple form

$$
\bar{\nabla}_{\mu} \bar{E}^{\mu}=0 .
$$

\section{References}

[1] Workshop on "Analog Models of General Relativity", Rio de Janeiro, October 2000, http://www.physics.wustl.edu//visser/Analog].

[2] L. Randall and R. Sundrum, "An alternative to compactification", Phys. Rev. Lett. 83 (1999) 46090, hep-th/9906064.

[3] B. Carter and J-P. Uzan, "Reflection symmetry breaking scenarios with minimal gauge form coupling in brane-world cosmology", Nuc. Phys. B (to appear), gr-qc/0101010. 
[4] R.A. Battye and B. Carter, "General junction conditions and minimal coupling in braneworld scenarios", Phys. Lett. B (to appear), hep-th/0101061.

[5] A. Kehagias and E. Kiritsis, "Mirage cosmology", JHEP 9911 (1999) 022, hep-th/9910174.

[6] D. Ida, "Brane-world cosmology", JHEP 0009 (2000) 014, gr-qc/9912002,

P. Krauss, "Dynamics of anti-de Sitter Domain Walls", JHEP 9912 (1999) 011, hep-th/9910149,

A.-C. Davis, S. Davis, W.B. Perkins, and I.R. Vernon, "Brane-world phenomenology and the $Z_{2}$ symmetry", Phys. Lett. B504 (2001) 254, hep-ph/0008132,

N. Deruelle and T. Dolezel, "Brane versus shell cosmologies in Einstein and EinsteinGauss-Bonnet theories", Phys. Rev. D62 (2000) 103502, gr-qc/0004021,

H. Stoica, H. Tye, and I. Wasserman, "Cosmology in the Randall-Sundrum brane-world scenario", Phys. Lett. B482 (2000) 205, hep-th/00004126],

W.B. Perkins, "Colliding bubble walls", Phys. Lett. B504 (2001) 28, gr-qc/0010053,

G. Kofinas, "New Perspectives on Moving Domain Walls in (A)dS $\mathrm{d}_{(5)}$ Space", hep-th/0103045.

[7] L. Anchordoqui, C. Nuñez, and K. Olsen, "Quantum cosmology and AdS/CFT", hep-th/0007064.

[8] P. Bowcock, C. Charmousis, and R. Gregory, "General brane cosmologies and their spacetime structure", Class. Quant. Grav. 17 (2000) 4745, [hep-th/0007177].

[9] R. Battye, B. Carter, A. Mennim, and J.-P. Uzan, "Einstein equations for an asymmetric brane-world", hep-th/0105091.

[10] T. Shiromizu, K. Maeda, and M. Sasaki, "The Einstein Equations on the 3-Brane World", Phys. Rev. D62 (2000) 024012, gr-qc/9910076.

[11] P. Binétruy, C. Deffayet, and D. Langlois, "Non-conventional cosmology from a brane universe", Nucl. Phys. B565 (2000) 269, hep-th/9905012.

[12] C.W. Misner, K.S. Thorne, and J.A. Wheeler, Gravitation (Freeman, San Francisco, 1973).

[13] R.A. Battye and B. Carter, "Gravitational perturbations of relativistic membranes and strings", Phys. Lett. B357 (1995) 29, hep-ph/9508300.

[14] T. Gherghetta and M. Shaposhnikov, "Localizing Gravity on a String-Like Defect in Six Dimensions", Phys. Rev. Lett. 85 (2000) 240, hep-th/0004014; T. Gherghetta, E. Roessl, and M. Shaposhnikov, "Living Inside a Hedgehog: Higher-dimensional Solutions that Localize Gravity", Phys. Lett. B491 (2000) 353, hep-th/0006251.

[15] B. Carter, "Essentials of classical brane dynamics", Proc. 2000 Peyresq meeting, Int. J. Theor. Phys. (to appear), gr-qc/0012036.

[16] J. Garriga and A. Vilenkin, "Perturbations on domain walls and string: a covariant theory", Phys. Rev. D44 (1991) 1007. 
[17] C. Will, "The Confrontation between General Relativity and Experiment", Living Reviews in Relativity 4 (2001) 2001-4 gr-qc/0103036.

[18] T. Damour and G. Esposito-Farèse, "Tensor-multi-scalar theories of gravitation", Class. Quant. Grav. 9 (1992) 2093.

[19] B. Carter, "Quasi gravity in branes", (in Proc. worshop on Analog Models for General Relativity, Rio, Oct., 2000) hep-th/0106037.

[20] G. Dvali, G. Gabadadze, and M. Shifman, "Ultralight Scalars and Spiral Galaxies", astro-ph/0102422.

[21] T. Damour and K. Nordtvedt, "Tensor-scalar cosmological models and their relaxation toward general relativity", Phys. Rv. D48 (1993) 3436.

[22] J.-P. Uzan and F. Bernardeau, "Lensing at cosmological scales: a test of higher dimensional gravity", hep-ph/0012011.

[23] J.O. Dickey et al, Science 265 (1994) 482.

[24] P. Droz-Vincent, "Mode solutions of the Klein-Gordon equation in warped spacetimes", Class. Quant. Grav. 18 (2001) 207, gr-qc/0003116. 